\title{
In situ monitoring of exopolymer- dependent Mn mineralization on bacterial surfaces
}

\author{
THAÏS COUASNON ${ }^{1,5}$, DAMIEN AlLOYEAU ${ }^{2}$, BENEDICTE
} MENEZ $^{1}$, FRANÇOIS GUYOT ${ }^{3}$, JEAN-MARC GHIGO ${ }^{4}$, LIANE G. BENNING ${ }^{5} \&$ ALEXANDRE GELABERT ${ }^{1}$

${ }^{1}$ Université de Paris, Institut de physique du globe de Paris, France.

${ }^{2}$ Université de Paris, Laboratoire Matériaux et Phénomènes Quantiques, Paris, France.

${ }^{3}$ IMPMC, Sorbonne Université, Paris, France.

${ }^{4}$ Unité de Génétique des Biofilms, Institut Pasteur, Paris, France.

${ }^{5}$ GFZ German Research Center for Geosciences, Telegrafenberg, 14473 Potsdam, Germany.

Bacterial biomineralization is a widespread process that largely impacts and has impacted the cycling of metals in present and past environments. Functionalized bacterial cell surfaces and exopolymers secreted in biofilms are suspected to play a key role as nucleation template initiating mineral formation. However, direct evidence under aqueous conditions of these mechanisms is hampered by technical challenges. Here, we present breakthrough in the use of liquid-cell scanning transmission electron microscopy to directly observe mineral growth on the surface of two Escherichia coli mutants forming distinct exopolymers. We use the incident electron beam to provoke and observe the precipitation of Mn-bearing minerals without any additional contrasting agent. Differences in precipitate morphology and distribution between the two mutants likely reflect differences in nucleation site density and accessibility associated with cell surfaces and exopolymers. This has strong environmental implications since biofilms structured by exopolymers are widespread and constitute the main form of microbial life on Earth. Besides, this study opens new perspectives on the use of incident electron beam in a liquid cell experiment to investigate the role of organics during the formation of a mineral phase involving redox sensitive metals. 